\title{
Severe Mitral Regurgitation Due to Pacing in Patient Post Aortic Valve Replacement (A Case Report)
}

\author{
Shailendra Kumar Motwani ${ }^{1}$, Vishnu Datt ${ }^{2}$, D. K. Tempe ${ }^{3}$ \\ ${ }^{1}$ National Board, G.B. Pant Hospital, New Delhi, India \\ ${ }^{2}$ Department of Cardiac Anesthesiology and Critical Care, G.B. Pant Hospital, New Delhi, India \\ ${ }^{3}$ Department of Cardiac Anesthesiology, G.B. Pant Hospital, New Delhi, India \\ Email: drshailendramotwani@gmail.com
}

How to cite this paper: Motwani, S.K., Datt, V. and Tempe, D.K. (2018) Severe Mitral Regurgitation Due to Pacing in Patient Post Aortic Valve Replacement (A Case Report). World Journal of Cardiovascular Surgery, 8, 22-27.

https://doi.org/10.4236/wjcs.2018.81003

Received: December 10, 2017

Accepted: January 19, 2018

Published: January 24, 2018

Copyright $\odot 2018$ by authors and Scientific Research Publishing Inc. This work is licensed under the Creative Commons Attribution International License (CC BY 4.0).

http://creativecommons.org/licenses/by/4.0/

\begin{abstract}
Epicardial Cardiac pacing may lead to severe Mitral Regurgitation by one of the following mechanisms: 1) Inappropriate Atrioventricular interval. 2) Myocardial ischemia due to fast heart rate may cause transient papillary muscle dysfunction. 3) Right Ventricular apical pacing may lead to Left Ventricular dyssynchrony in the presence of optimal Atrioventricular synchrony. Acute severe Mitral Regurgitation leading to acute severe hemodynamic deterioration is a reported complication of Permanent Pacemaker insertion. Our case demonstrated acute severe MR as a consequence of RV Pacing leading to acute hemodynamic deterioration, which was relieved on withdrawal of pacing. Left Ventricular dyssynchrony can be relieved by reducing the peacemaker rate or changing to biventricular pacing, this reduces the severity of Mitral Regurgitation and improves the hemodynamics due to simultaneous activation of left and right ventricles. This case illustrates the acute and potentially dramatic effects of intra-Left Ventricular dyssynchrony upon Mitral Valve function. Informed consent was obtained from the patient to report the case. Thus right ventricular pacing can cause left ventricular dyssynchrony leading to worsening of Mitral Regurgitation. It is important to pay attention to mode of pacing when evaluating Mitral Regurgitation in patients with Right Ventricular pacemaker and unstable hemodynamics after initiation of pacing.
\end{abstract}

\section{Keywords}

Epicardial Pacing, Mitral Regurgitation, Dyssynchrony, Hemodynamics, Cardiopulmonary Bypass, Atrioventricular Synchrony, Atrioventricular Interval 


\section{Introduction}

Functional mitral regurgitation (MR) is characterized by the absence of structural abnormalities of the mitral valve leaflets, chordae, and papillary muscles. Left intraventricular conduction delay or its equivalent in the form of right ventricular (RV) pacing may precipitate or aggravate varying degrees of functional Mitral Regurgitation. Severe pacemaker-induced Mitral Regurgitation may be partially or largely reversible by restoration of LV synchrony with LV/biventricular pacing and in the occasional patient simply by programming the pacemaker to a low rate to permit the emergence of spontaneous beats thereby eliminating LV dyssynchrony [1].

The following case report demonstrates the RV pacing induced Mitral Regurgitation in the peri-operative period.

\section{Case Summary}

A case of 40 years old male who presented with NYHA Class III symptoms.

Transthoracic echocardiography revealed RHD with severe aortic stenosis severe AS with peak gradient of $70 \mathrm{mmHg}$ and mean gradient of $46 \mathrm{~mm} \cdot \mathrm{Hg}$, Mild MR, EF $60 \%$.

The patient was scheduled to undergo Aortic Valve Replacement.

After standard cardiac induction with balanced opioid based general anesthesia technique, the patient underwent aortic valve replacement on standard cardiopulmonary bypass with mild hypothermia $\left(32^{\circ} \mathrm{C}\right)$ using antegrade Potassium Enriched Blood Cold Cardioplegia. The aortic valve was replaced with $20 \mathrm{Med}-$ tronic metallic prosthetic aortic valve. The total bypass time was 81 minutes and total aortic cross clamp time (ischemia time) was 50 minutes.

Post AVR there was a conduction block so weaning from CPB was commenced with direct epicardial pacing at the rate of 100 beats/min with pacemaker in Asynchronous Ventriculo-Ventricular Inhibition mode (VVI) mode and the patient was weaned off bypass using high dose inotropes (Dobutamine 8 $\mathrm{mcg} / \mathrm{kg} / \mathrm{min}$; Adrenaline $0.1 \mathrm{mcg} / \mathrm{kg} / \mathrm{min}$; NTG1 mcg $/ \mathrm{kg} / \mathrm{min}$ ).

Immediately coming off bypass Transoesophageal Echocardiography revealed there was severe mitral regurgitation seen on TEE (Figure 1). There was hemodynamic deterioration even with the use of higher doses of inotropes (BP 65/40 $\mathrm{mm} \cdot \mathrm{Hg}$; CVP $14 \mathrm{~cm} \mathrm{H}_{2} \mathrm{O}$ ).

There was discussion for resuming cardiopulmonary bypass and performing mitral valve replacement in view of severe MR.

Meanwhile the heart rate and rhythm improved and pacing was stopped due to sinus rhythm of 90 beats/min. There was a sudden improvement in the hemodynamics of the patient improved (BP $106 / 70 \mathrm{~mm} \cdot \mathrm{Hg}$; $\mathrm{CVP} 5 \mathrm{~cm} \mathrm{H}_{2} \mathrm{O}$ ) with sinus rhythm at rate of 90 beats/min).

Repeat TEE revealed no significant mitral regurgitation (Figure 2). So Mitral valve replacement was deferred. The patient remained stable in the postoperative period. 


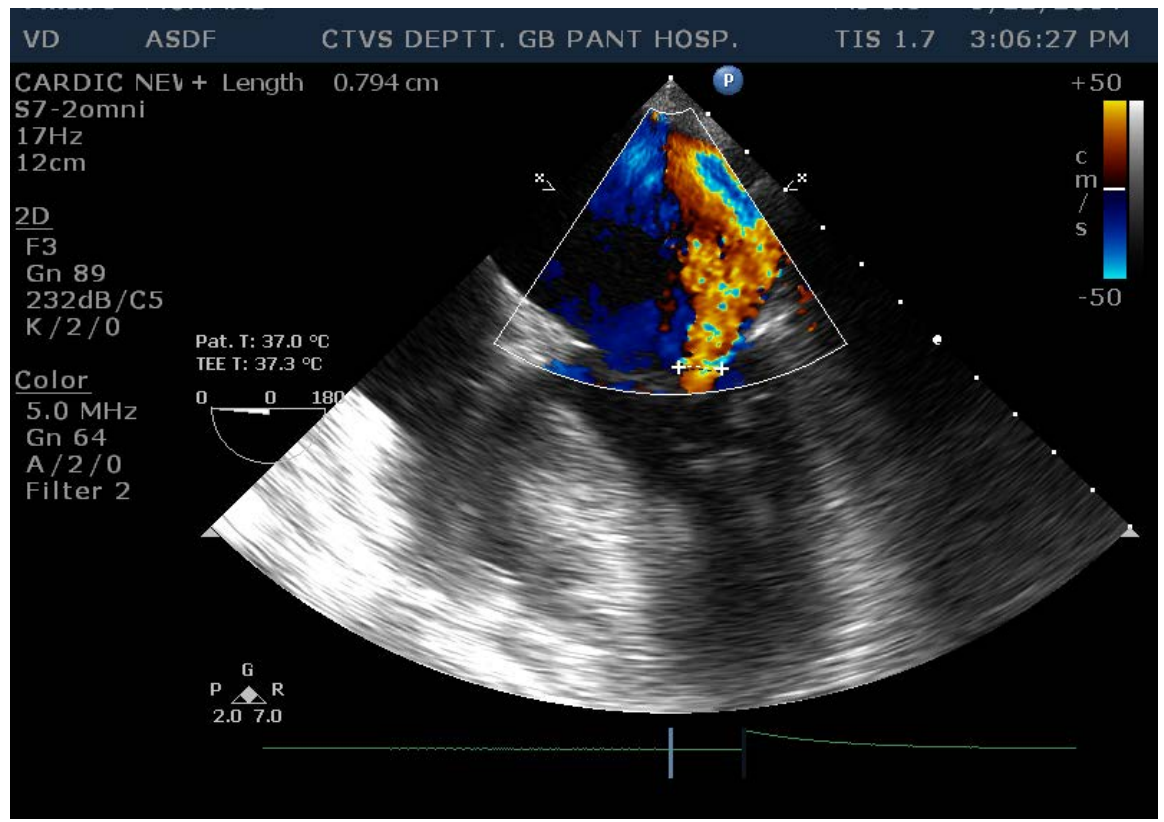

Figure 1. RV pacing induced MR.

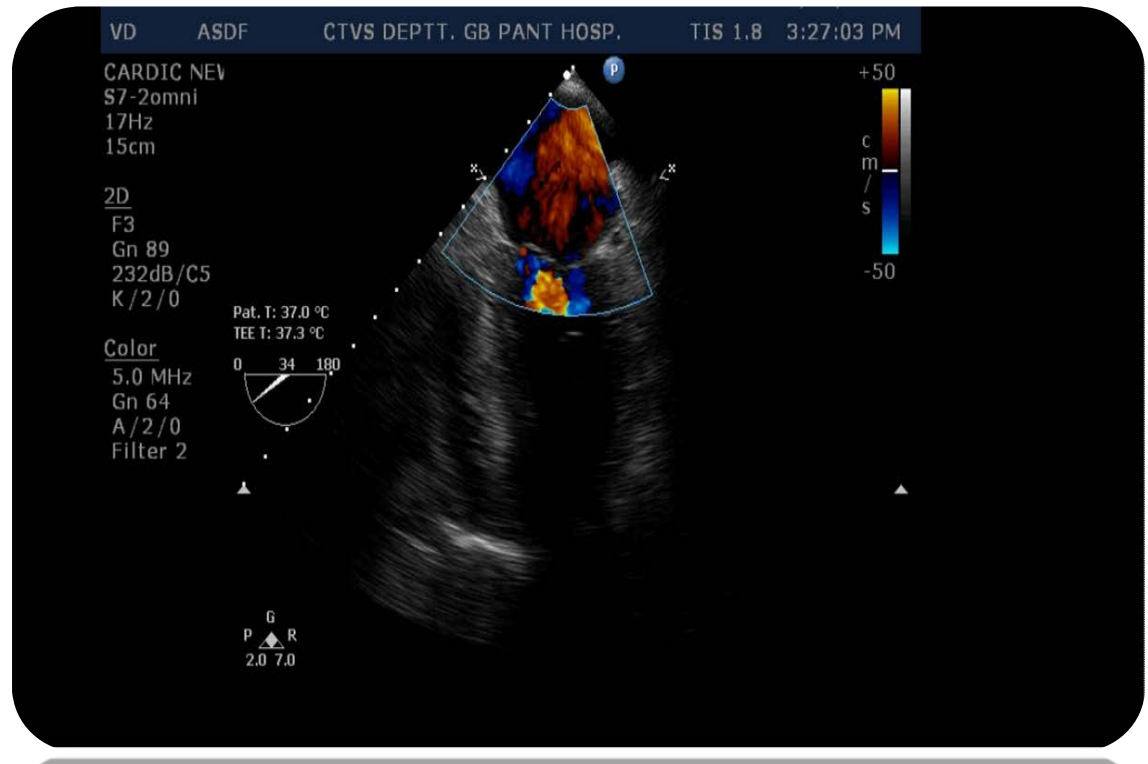

Figure 2. No MR after removal of Pacing.

\section{Discussion}

Acute severe MR due to cardiac Pacing may be caused by one of the following mechanisms:

1) Inappropriate atrioventricular conduction interval [2] [3].

2) Myocardial ischemia induced by fast rate leading to papillary muscle dysfunction [4] [5].

3) RV apical pacing may lead to LV dyssynchrony.

This case report demonstrates the acute and potentially dramatic effects of intraventricular dyssynchrony upon MV competence. 
Secondly it highlights the deleterious effects of $R V$ apical pacing on intraventricular synchrony and the severity of MR.

Thirdly Atrioventricular ( $A V)$ dyssynchrony during VVI pacing may also have contributed to MR.

This case demonstrated that during ventricular pacing there was severe MR that reduced markedly after discontinuation of pacing.

LV dyssynchrony as a cause of MR was demonstrated by Maurer et al. [6] due to RV apical pacing, and basal LV pacing via the coronary sinus. The MR was severe during RV pacing and relatively mild during LV pacing and the MR was relieved with sinus rhythm.

DDD pacemaker insertion to improve heart failure symptoms in Hypertrophic Obstructive Cardiomyopathy patients can be associated with systolic mitral regurgitation as the cause of failure in DDD pacing therapy [7].

De Guillebon et al. [8] have reported many cases of pacemaker-induced, functional MR and congestive heart failure several months after pacemaker implantation.

Functional mitral regurgitation may occur in patients with dilated cardiomyopathy and CHF due to Left intraventricular conduction delay or its equivalent Right Ventricular (RV) pacing [9] [10] [11].

\section{Treatment of acute severe $M R$ after $R V$ Pacing.}

Severe pacemaker-induced MR may be relieved by restoration of $L V$ synchrony with LV/biventricular pacing or simply by programming the pacemaker to a low rate to permit the emergence of spontaneous beats thereby eliminating LV dyssynchrony [12] [13].

Biventricular pacing leads to improvement in MR in CHF patient with a narrow QRS complex and echocardiographic evidence of LV dyssynchrony. This illustrates the primary role of mechanical rather than electrical dyssynchrony in the genesis of functional MR in the absence of a visible intraventricular conduction disorder [14] [15] [16].

There is variable improvement in MR through reverse LV remodeling and changes in ventricular geometry which leads to more complete MV closure [17].

Pacemaker induced functional $M R$ which was earlier considered as a part of the pacemaker syndrome, is currently defined as the clinical consequence of suboptimal AV synchrony [18].

Thus pacemaker induced MR and hemodynamic deterioration can be managed by restoration of $L V$ synchrony by:

$>$ Resynchronisation and changing to Biventricular Pacing.

$>$ Reprogramming the pacemaker to a low rate which leads to the emergence of spontaneous beats thereby eliminating LV dyssynchrony.

$>$ RVOT pacing improves ventricular dyssynchrony, and avoids the need for upgrading to a biventricular device in a patient with no previous symptoms of heart failure and with preserved left ventricular function.

Our case demonstrated that RV pacing can lead to acute severe MR, and se- 
vere hemodynamic deterioration and this severe $M R$ was reduced markedly when not paced.

The severity of MR can be significantly reduced by Lowering the pacemaker rate or upgrading to biventricular pacing by allowing simultaneous activation of the left and right ventricles, thus avoids LV dyssynchrony.

\section{Conclusions}

Right ventricular pacing can cause left ventricular dyssynchrony leading to severe functional MR.

It is important to pay attention to mode of pacing when evaluating MR in patients with Right ventricular pacemaker and unstable hemodynamics after initiation of pacing.

\section{References}

[1] Barold, S.S. and Ovsyshcher, I.E. (2005) Pacemaker-Induced Mitral Regurgitation. Pacing and Clinical Electrophysiology, 28, 357-360. https://doi.org/10.1111/j.1540-8159.2005.09486.x

[2] Haas, J.M. and Strait, G.B. (1974) Pacemaker-Induced Cardiovascular Failure. Hemodynamic and Angiographic Observations. American Journal of Cardiology, 33, 295-299. https://doi.org/10.1016/0002-9149(74)90293-8

[3] Berglund, H., Nishioka, T., Hackner, E., Kim, C.J., Luo, H., Fontana, G. and Siegel, R.J. (1996) Ventricular Pacing: A Cause of Reversible Severe Mitral Regurgitation. American Heart Journal, 135, 1035-1037. https://doi.org/10.1016/S0002-8703(96)90191-X

[4] Kamp, O., de Cock, C.C., van Eenige, M.J. and Visser, C.A. (1994) Influence of Pacing-Induced Myocardial Ischemia on Left Atrial Regurgitant Jet: A Transesophageal Echocardiographic Study. Journal of the American College of Cardiology, 23, 1584-1591. https://doi.org/10.1016/0735-1097(94)90660-2

[5] Myrmel, T., Lai, D.T., Lo, S., Timek, T.A., Liang, D., Miller, D.C., Ingels, N.B. and Jr, Daughters, G.T. (2002) Ischemia-Induced Malcoaptation of Scallops within the Posterior Mitral Leaflet. The Journal of Heart Valve Disease, 11, 823-829.

[6] Maurer, G., Torres, M.A.R., Corday, E., Haendchen, R.V. and Meerbaum, S. (1984) Two-Dimensional Echocardiographic Contrast Assessment of Pacing-Induced Mitral Regurgitation: Relation to Altered Regional Left Ventricular Function. Journal of the American College of Cardiology, 3, 986-991. https://doi.org/10.1016/S0735-1097(84)80357-5

[7] Kataoka, H. (2006) Pacemaker-Induced Mitral Regurgitation as a Cause of Refractory Congestive Heart Failure during Pacing Therapy in a Patient with Hypertrophic Obstructive Cardiomyopathy. Congestive Heart Failure, 12, 112-115. https://doi.org/10.1111/j.1527-5299.2006.04768.x

[8] de Guillebon, M. (2007) Regression of Mitral Regurgitation after Cardiac Resynchronization Therapy in an Adult with Preserved Left Ventricular Function and Right Ventricular Pacing. Europace, 9, 768-769. https://doi.org/10.1093/europace/eum069

[9] Littmann, L. and Symanski, J.D. (2000) Hemodynamic Implications of Left Bundle Branch Block. Journal of Electrocardiology, 33, 115-121.

https://doi.org/10.1054/jelc.2000.20330 
[10] Kass, D.A. (2004) Pathophysiology of Cardiac Dyssynchrony and Resynchronization. In: Ellenbogen, K.A., Kay, G.N. and Wilkoff, B.L., Eds., Device Therapy for Congestive Heart Failure, Saunders, Philadelphia, 27-46.

[11] Erlebacher, J.A. and Barbarash, S. (2001) Intraventricular Conduction Delay and Functional Mitral Regurgitation. American Journal of Cardiology, 88, 83-86. https://doi.org/10.1016/S0002-9149(01)01595-8

[12] Cannan, C.R., Higano, S.T. and Holmes, D.R. (1997) Pacemaker Induced Mitral Regurgitation: An Alternative Form of Pacemaker Syndrome. PACE, 20, 735-738. https://doi.org/10.1111/j.1540-8159.1997.tb03895.x

[13] Janousek, J., Tomek, V., Chaloupecky, V. and Gebauer, R.A. (2004) Dilated Cardiomyopathy Associated with Dual-Chamber Pacing in Infants: Improvement through Either Left Ventricular Cardiac Resynchronization or Programming the Pacemaker off Allowing Intrinsic Normal Conduction. Journal of Cardiovascular Electrophysiology, 15, 470-474. https://doi.org/10.1046/j.1540-8167.2004.03481.x

[14] Achilli, A., Sassara, M., Ficili, S., Pontillo, D., Achilli, P., Alessi, C., De Spirito, S., Guerra, R., Patruno, N. and Serra, F. (2003) Long-Term Effectiveness of Cardiac Resynchronization Therapy in Patients with Refractory Heart Failure and "Narrow" QRS. Journal of the American College of Cardiology, 42, 2117-2124. https://doi.org/10.1016/j.jacc.2003.08.024

[15] Gasparini, M., Mantica, M., Galimberti, P., Marconi, M., Genovese, L., Faletra, F., Simonini, S., Klersy, C., Coates, R. and Gronda, E. (2003) Beneficial Effects of Biventricular Pacing in Patients with a "Narrow" QRS. PACE, 26, 169-174. https://doi.org/10.1046/j.1460-9592.2003.00010.x

[16] Turner, M.S., Bleasdale, R.A., Mumford, C.E., Frenneaux, M.P. and Morris-Thurgood, J.A. (2004) Left Ventricular Pacing Improves Haemodynamic Variables in Patients with Heart Failure with a Normal QRS Duration. Heart, 90, 502-505. https://doi.org/10.1136/hrt.2003.011759

[17] Erol-Yilmaz, A., Tukkie, R., Schrama, T.A., Romkes, H.J. and Wilde, A.A. (2002) Reverse Remodelling of Dilated Left Sided Cardiomyopathy after Upgrading from VVIR to VVIR Biventricular Pacing. Europace, 4, 445-449. https://doi.org/10.1053/eupc.2002.0252

[18] Ellenbogen, K.A., Gilligan, D.M., Wood, M.A., et al. (1997) The Pacemaker Syndrome-A Matter of Definition. American Journal of Cardiology, 79, 1226-1229. https://doi.org/10.1016/S0002-9149(97)00085-4 